\title{
Theoretical advances on Economic Model Predictive Control with time-varying costs
}

\author{
David Angeli ${ }^{\mathrm{a}}$ Alessandro Casavola ${ }^{\mathrm{b}}$ Francesco Tedesco ${ }^{\mathrm{b}}$ \\ ${ }^{a}$ Department of Electrical and Electronic Engineering, Imperial College London, SW7 2AZ, U.K., and also with the \\ Department of Information Engineering, University of Florence, Italy \\ d.angeli@imperial.ac.uk \\ ${ }^{\mathrm{b}}$ Departement of Information Engineering, Modeling, Electronic and Systems - University of Calabria, Rende(CS), Italy \\ \{alessandro.casavola, francesco.tedesco\}@unical.it
}

\begin{abstract}
Economic Model Predictive Control is a technique for optimization of economic revenues arising from controllod dynamical processes that has estabilished itself as a variant of standard Tracking Model Predictive Control. It departs from the latter in that arbitrary cost functions are allowed in the formulation of the stage cost. This paper takes a further step in expanding the applicability of Economic Model Predictive Control by illustrating how the paradigm can be adapted in order to accomodate time-varying or parameter-varying costs.
\end{abstract}

Key words:

\section{Introduction and motivations}

Model Predictive Control is a model-based control design technique for MIMO systems subject to input and state constraints. In its classical formulation it allows to formulate general tracking problems for nonlinear and/or linear systems by taking into account modelbased predictions throughout a finite control horizon and setting up the control selection algorithm as an on-line optimization problem where the adopted cost function is a measure of the discrepancy of the predicted trajectory with respect to the desired set-point signal.

In recent years, Economic MPC has emerged as a variant of Model Predictive Control where the primary control task is profitability enhancement, rather than minimization of a tracking error. From a mathematical perspective, this amounts to considering cost functionals, defined over a typically finite control horizon, which can take arbitrary shape, rather than being limited to some (positive definite) distance function from a set-point of interest. In particular, for nonlinear systems and/or non convex cost functionals, profitability may be maximal away from equilibrium states and this may in turn lead to complex regimes of operation or transient behaviours which may exhibit highly nonlinear features, such as asymmetry with respect to initial conditions or slow and highly oscillatory decays.

Motivated by applications in areas where price variations are comparable in speed with process dynamics, the case of time-varying costs or parameter-varying costs were recently explored in Ellis \& Christofides (2014). The method developed is a Lyapunov-based Economic MPC scheme which allows to guarantee boundedness of solutions as well as constraints satisfaction while attempting to optimize a time-varying cost functional.

As a matter of fact, in recent years, many efforts have been devoted to investigate Economic MPC variants allowing time-varying costs in several domains of application: management of energy in buildings (Touretzky \& Baldea (2014); Ma et al. (2014)), control of chemical plants (Ellis \& Christofides (2014)) and supervision of distribution networks, such as water networks (Grosso et al. (2014)), power grids (Hovgaard et al. (2010); Cole et al. (2014); Adeodu \& Chmielewski (2013), ) gas networks, etc (Gopalakrishnan \& Biegler (2013)). Other Economic MPC approaches dealing either time-varying cost or cyclic plant operations from a theoretical perspective can be found in Ferramosca et al. (2014); Limon et al. (2014); Huang et al. (2012). Indeed, the extension of Economic Model Predictive Control to encompass cost variability appears to be a natural question, both from 
a practical and theoretical perspective, and conceptually similar to the traditional departure from set-point tracking towards tracking of more general reference trajectories.

Two recent papers, Angeli et al. (2015b) and Angeli et al. (2015a), have attempted to extend the Economic Model Predictive Control analysis framework of Angeli et al. (2012) to this set-up. Hereby we recall the control algorithms and the main results of Angeli et al. (2015b) and Angeli et al. (2015a) while providing a comparison of their main merits and limitations, together with a simulated case study where both approaches are tested and evaluated against each other. This manuscript is an extended version of Angeli et al. (2015c) suitably edited for this special issue.

\section{Preliminaries and problem set-up}

The basic formulation of Economic Model Predictive Control deals with discrete-time systems of the following form:

$$
x(t+1)=f(x(t), u(t)), \quad x(0)=x_{0}
$$

with $t \in \mathbb{N}$, state variable $x \in \mathcal{X} \subset \mathbb{R}^{n}$, control input $u \in$ $\mathcal{U} \subset \mathbb{R}^{m}$ and continuous state-transition map $f: \mathcal{X} \times$ $\mathcal{U} \rightarrow \mathcal{X}$. Additionally, system's evolutions are subject to pointwise-in-time constraints involving both states and input variables,

$$
(x(t), u(t)) \subset \mathcal{Z} \quad \forall t \geq 0
$$

for some compact set $\mathcal{Z} \subset \mathcal{X} \times \mathcal{U}$. The control task is to fulfill constraints (2) while, at the same time, minimizing a cost functional defined integrating over time an instantaneous (continuous) stage cost $\ell$ defined as:

$$
\ell(x, u): \mathcal{X} \times \mathcal{U} \rightarrow \mathbb{R}
$$

For the case of Tracking Model Predictive Control, the stage cost $\ell$ typically takes the form of a quadratic function $x^{\prime} Q x+u^{\prime} R u$, or a shifted version of this, if an equilibrium either than 0 is the desired target state.

In Economic Model Predictive Control, on the contrary, $\ell$ may take an arbitrary shape, and this, in turn, can affect considerably the optimal regimes of operations for the system. Notice that the basic formulation of Economic Model Predictive Control only entails timeinvariant "ingredients", viz. dynamics, (1), operational constraints, (2), and operational costs, (3). While it is conceivable to allow all of them to be time-varying, we argue that, in many applications of interest, dynamics are in fact time-invariant, while the only significant source of variability happens at the level of both cost and constraints.

This is because a plant often operates in a manner that does not change in time, apart from deteriorating phenomena that are normally much slower than the timescales of interest. In this respect, only the environment the plant is interacting with may experience faster and significant variations. Moreover, if we are talking about an 'economic environment', rather than a physical one, time-varying constraints are typically not safety critical, and can often be modeled as soft constraints, that is, as suitable cost penalties incurred only in case of constraints violation.

These considerations allow to remarkably simplify the set-up of a time-varying Economic Model Predictive Control scheme and the associated analysis. They allow, in fact, to avoid feasibility issues and associated technical complications that are known to occur whenever time-variability affects constraints or dynamics. On the grounds of such considerations, we consider next two possible modifications of (3), in order to accommodate time-varying or parameter-varying stage costs. Namely, we allow $\ell$ to directly depend on time:

$$
\ell(t, x, u): \mathbb{N} \times \mathcal{X} \times \mathcal{U} \rightarrow \mathbb{R}
$$

or indirectly through a time-varying parameter $\theta$ taking up finitely many values in $\Theta:=\left\{\theta_{1}, \theta_{2}, \ldots, \theta_{N}\right\}$ :

$$
\tilde{\ell}(\theta, x, u): \Theta \times \mathcal{X} \times \mathcal{U} \rightarrow \mathbb{R}
$$

As expected, we are going to formulate the control selection policy as the solution of an associated optimization problem to be performed on-line at each sampling time on the basis of the current knowledge of state and future predictions of both systems trajectories and stage costs variations.

Notice that, also in the case of parameter-varying stage costs, once the time evolution of the parameter $\theta$ is assigned, one may define a corresponding time-varying cost simply by composition of the functions $\tilde{\ell}$ and $\theta(t)$, by letting:

$$
\ell(t, x, u):=\tilde{\ell}(\theta(t), x, u) .
$$

This notation allows to formulate a unified cost functional for both cases, by considering:

$$
J_{H}(t, \mathbf{x}, \mathbf{u})=\sum_{k=0}^{H-1} \ell(t+k, x(k), u(k))
$$

where $\mathbf{x}:=[x(0), x(1), \ldots, x(H-1), x(H)]$ denotes the sequence of predicted states, $\mathbf{u}=[u(0), \ldots, u(H-1)]$ that of predicted controls and $H$ denotes the prediction horizon. In both scenarios, the input $u$ is selected at each 
sampling time $t$ by finding a solution to the following optimization problem:

$$
\begin{aligned}
J_{H}^{\star}\left(t, x(t), x_{F}(t)\right):= & \min _{\mathbf{z}, \mathbf{v}} J_{H}(t, \mathbf{z}, \mathbf{v}) \\
& \text { subject to: } \\
& z(k+1)=f(z(k), v(k)), \\
& (z(k), v(k)) \in \mathcal{Z}, k=0, \ldots, H-1 \\
& z(H)=x_{F}(t) \\
& z(0)=x(t)
\end{aligned}
$$

and letting $u(t)$ correspond to the first input value of any optimal sequence $\mathbf{v}^{\star}$ solution of (8). Notice that, while continuity and compactness considerations guarantee existence of an optimal solution, this might in general be non unique.

The terminal equality constraint, often used in MPC as a means to achieve recursive feasibility (and possibly stabiliy), is specified in (8) as a function of a suitably defined feasible trajectory $x_{F}(t)$. Indeed, $x_{F}(t)$ can be selected according to different criteria. However, existence of an input $u_{F}(t)$ fulfilling:

(1) $x_{F}(t+1)=f\left(x_{F}(t), u_{F}(t)\right) \quad \forall t \geq 0$,

(2) $\left(x_{F}(t), u_{F}(t)\right) \in \mathcal{Z} \quad \forall t \geq 0$,

is crucial to the following developments. Defining the sequence of terminal constraints according to a feasible solution, in fact, allows to easily achieve two important goals: recursive feasibility and guaranteed optimal performance. We recall next one of the technical Lemmas in Angeli et al. (2015a).

Lemma 1 Consider system (1), controlled by the following time-varying state-feedback,

$$
u(t)=\mathbf{v}_{0}^{\star}(t, x(t))
$$

where $\mathbf{v}^{\star}(t, x(t))$ denotes the solution, at each time $t$, of the optimization problem (8). Then, if a feasible solution exists at time 0 , problem (8) is feasible at each subsequent time $t$. Moreover, the asymptotic average cost of closedloop trajectories is bounded from above as follows:

$$
\begin{aligned}
& \limsup _{\tau \rightarrow+\infty} \frac{\sum_{t=0}^{\tau-1} \ell(t, x(t), u(t))}{\tau} \\
& \leq \limsup _{\tau \rightarrow+\infty} \frac{\sum_{t=0}^{\tau-1} \ell\left(t+H, x_{F}(t), u_{F}(t)\right)}{\tau} .
\end{aligned}
$$

Proof: Let $\mathbf{z}^{\star}(t, x(t))$ and $\mathbf{v}^{\star}(t, x(t))$ denote the optimal state and control sequences for problem (8) at time $t$. Then, at time $t+1$, the shifted state and control sequences $\tilde{\mathbf{z}}=\left[\mathbf{z}_{1: H}^{\star}(t, x(t)), x_{F}(t+1)\right]$, $\tilde{\mathbf{v}}=\left[\mathbf{v}^{\star}(t, x(t)), u_{F}(t)\right]$ are also feasible for problem (8) computed on the state $x(t+1)=f(x(t), u(t))$. Hence, recursive feasibility can be proved by induction provided that $x(0)$ is a feasible solution for (8) at time $t=0$. Furthermore, for the cost functional, we see that

$$
\begin{aligned}
& J_{H}^{\star}\left(t+1, x(t+1), x_{F}(t+1)\right) \leq J_{H}(t+1, \tilde{\mathbf{z}}, \tilde{\mathbf{v}}) \\
& =J_{H}\left(t, \mathbf{z}^{\star}(t, x(t)), \mathbf{v}^{\star}(t, x(t))\right)-\ell(t, x(t), u(t)) \\
& \quad+\ell\left(t+H, x_{F}(t), u_{F}(t)\right) \\
& =J_{H}^{\star}\left(t, x(t), x_{F}(t)\right)-\ell(t, x(t), u(t)) \\
& \quad+\ell\left(t+H, x_{F}(t), u_{F}(t)\right)
\end{aligned}
$$

holds regardless of $t \in \mathbb{N}$. Moreover, by (9) it follows:

$$
\begin{aligned}
& J_{H}^{\star}\left(\tau, x(\tau), x_{F}(\tau)\right)-J_{H}^{\star}\left(0, x(0), x_{F}(0)\right) \\
& =\sum_{t=0}^{\tau-1} J_{H}^{\star}\left(t+1, x(t+1), x_{F}(t+1)\right)-J_{H}^{\star}\left(t, x(t), x_{F}(t)\right) \\
& \leq \sum_{t=0}^{\tau-1}-\ell(t, x(t), u(t))+\ell\left(t+H, x_{F}(t), u_{F}(t)\right)
\end{aligned}
$$

Finally, by dividing the cost by $\tau$ and letting $\tau \rightarrow+\infty$, it is found that:

$$
\begin{aligned}
0 & =\liminf _{\tau \rightarrow+\infty} \frac{J_{H}^{\star}\left(\tau, x(\tau), x_{F}(\tau)\right)-J_{H}^{\star}\left(0, x(0), x_{F}(0)\right)}{\tau} \\
& \leq \liminf _{\tau \rightarrow+\infty} \frac{\sum_{t=0}^{\tau-1}-\ell(t, x(t), u(t))+\ell\left(t+H, x_{F}(t), u_{F}(t)\right)}{\tau}
\end{aligned}
$$

where the first equality follows from the uniform boundedness of $J_{H}^{\star}$ on the compact set $\mathcal{Z}$.

In words the Lemma states that closed-loop solutions have an asymptotic average cost that is never worse than that of the feasible solution adopted as a terminal constraint. It is therefore desirable to select $x_{F}(t)$ in order to minimize its average asymptotic cost. In the next two Sections we discuss criteria for the selection of $x_{F}(t)$, both in the case of time-varying and parameter-varying stage costs.

\section{EMPC with time-varying stage costs}

In order to select a suitable terminal constraint for the optimization problem (8), we need to formulate extra assumptions on the variability that $\ell(t, x, u)$ may exhibit. Two cases are considered:

(1) Periodic stage cost: $\exists T>0: \quad \ell(t+T, x, u)=$ $\ell(t, x, u) \quad \forall t \in \mathbb{N}$ 
(2) Stage cost admitting asymptotic average:

$$
\bar{\ell}(x, u)=\lim _{T \rightarrow+\infty} \frac{\sum_{t=0}^{T-1} \ell(t, x, u)}{T} .
$$

Clearly, a periodic stage cost admits an asymptotic average; however, the converse implication need not hold. It is worth pointing out that $\bar{\ell}$ is defined for frozen state and input values. In this respect, its value as a function of $x$ and $u$ need not correspond to some form of average cost experienced along solutions of the system. All phase information (concerning variations of the cost experienced at different times for any fixed $(x, u)$ pair) is, in fact, lost. For the simplest case of $T$-periodic stage costs, one may define the best periodic solution of period $T$ as follows:

$$
\begin{aligned}
\ell_{T}^{\star}= & \min _{\mathbf{z}, \mathbf{v}} J_{T}(t, \mathbf{z}, \mathbf{v}) / T \\
& \text { subject to: } \\
& z(k+1)=f(z(k), v(k)), \quad k=0, \ldots, T-1 \\
& z(T)=z(0) \\
& (z(k), v(k)) \in \mathcal{Z}, \quad k=0, \ldots, T-1 .
\end{aligned}
$$

Letting $\mathbf{z}^{\star}, \mathbf{v}^{\star}$ denote the optimal solutions of (12), we may define $x_{F}(t):=\mathbf{z}^{\star}(t+H \bmod T)$ and $u_{F}(t):=$ $\mathbf{v}^{\star}(t+H \bmod T)$. This leads to a periodic terminal solution $x_{F}, u_{F}$ which has the best cost among all feasible solutions of period $T$. For more general stage costs, such as those admitting a well defined asymptotic average, we may define the best $Q$ periodic solution computed on averaged costs as follows:

$$
\begin{aligned}
\bar{\ell}_{Q}^{\star}= & \min _{\mathbf{z}, \mathbf{v}} \bar{J}_{Q}(\mathbf{z}, \mathbf{v}) / Q \\
& \text { subject to: } \\
& z(k+1)=f(z(k), v(k)), \quad k=0, \ldots, Q-1 \\
& z(Q)=z(0) \\
& (z(k), v(k)) \in \mathcal{Z}, \quad k=0, \ldots, Q-1 .
\end{aligned}
$$

where the averaged cost functional $\bar{J}_{Q}$ is defined as:

$$
\bar{J}_{Q}(\mathbf{x}, \mathbf{u})=\sum_{k=0}^{Q-1} \bar{\ell}(x(k), u(k)) .
$$

Similarly, letting $\mathbf{z}^{\star}, \mathbf{v}^{\star}$ denote the optimal solutions of (13), we may define $x_{F}(t):=\mathbf{z}^{\star}(t \bmod Q)$ and $u_{F}(t):=$ $\mathbf{v}^{\star}(t \bmod Q)$. Proposition 1 in Angeli et al. (2015b) states that, for periodic stage costs, the inequality $\ell_{T}^{\star} \leq$ $\bar{\ell}_{T}^{\star}$ holds; as intuitive, periodicity of stage-costs allows selection of a better terminal constraint than what can be achieved by only considering averaged stage costs $\bar{\ell}$. On the contrary, if allowing periods $Q \neq T$, no simple inequality between $\ell_{T}^{\star}$ and $\ell_{Q}^{\star}$ can be claimed in general (see Example 1 in Angeli et al. (2015b)). Furthermore, if the averaged stage-cost can be achieved also by averaging the stage cost every $Q$ sampling times, then the average cost of $x_{F}(t)$ as defined above, can be related to $\bar{\ell}_{Q}^{\star}$. To this end the following assumption is needed:

Assumption 1 Q-steps sampling averaged cost is $t$ independent and equals $\bar{\ell}(x, u)$ :

$$
\bar{\ell}(x, u)=\lim _{L \rightarrow+\infty} \frac{\sum_{k=0}^{L-1} \ell(t+k Q, x, u)}{L} .
$$

Notice that this is fulfilled, for instance, if $\ell$ is periodic of period $T$ and, at the same time, $T$ and $Q$ are relatively prime numbers.

The following Lemma allows to relate the performance bound provided by Lemma 1 with the cost of the best $Q$-periodic solution computed on averaged stage-costs.

Lemma 2 Let $\ell(t, x, u)$ admits $t$ independent $Q$-steps averaged cost. Then, denoting $x_{F}(t)$ and $u_{F}(t)$ the best $Q$-periodic solution of (13) and associated input, the following holds:

$$
\lim _{\tau \rightarrow+\infty} \frac{\sum_{t=0}^{\tau-1} \ell\left(t+H, x_{F}(t), u_{F}(t)\right)}{\tau}=\bar{\ell}_{Q}^{\star} .
$$

Proof: The Lemma is proved by performing the following calculations:

$$
\begin{aligned}
& \lim _{\tau \rightarrow+\infty} \frac{\sum_{t=0}^{\tau-1} \ell\left(t+H, x_{F}(t), u_{F}(t)\right)}{\tau} \\
= & \lim _{\tau \rightarrow+\infty} \frac{\sum_{t=0}^{\tau-1} \ell\left(t+H, \mathbf{z}^{\star}(t \bmod Q), \mathbf{v}^{\star}(t \bmod Q)\right)}{\tau} \\
= & \lim _{\tau \rightarrow+\infty} \sum_{i=0}^{Q-1} \frac{\sum_{k=0}^{\left\lfloor\frac{\tau-1-i}{Q}\right\rfloor} \ell\left(k Q+H+i, \mathbf{x}^{\star}(i), \mathbf{u}^{\star}(i)\right)}{\tau} \\
= & \frac{\sum_{i=0}^{Q-1} \frac{\bar{\ell}\left(\mathbf{x}^{\star}(i), \mathbf{u}^{\star}(i)\right)}{Q}=\bar{\ell}_{Q}^{\star},}{Q}
\end{aligned}
$$

where the equalities hold true thanks to Assumption 1 and can be justified by denoting $\tau-1=S Q+(\tau-$ 1) $\bmod Q, S \in \mathbb{N}$ and taking into account the following 
intermediate steps:

$$
\begin{aligned}
& \lim _{\tau \rightarrow+\infty} \frac{\sum_{k=0}^{\left\lfloor\frac{\tau-1-i}{Q}\right\rfloor} \ell\left(k Q+H+i, \mathbf{x}^{\star}(i), \mathbf{u}^{\star}(i)\right)}{\tau} \\
= & \lim _{S \rightarrow+\infty} \frac{\sum_{k=0}^{S+\left\lfloor\frac{(\tau-1) \bmod }{Q-i}\right\rfloor} \ell\left(k Q+H+i, \mathbf{x}^{\star}(i), \mathbf{u}^{\star}(i)\right)}{S Q+(\tau-1) \bmod Q} \\
= & \lim _{S \rightarrow+\infty} \frac{\sum_{k=0}^{S-1} \ell\left(k Q+H+i, \mathbf{x}^{\star}(i), \mathbf{u}^{\star}(i)\right)}{S Q+(\tau-1) \bmod Q} \\
& +\lim _{S \rightarrow+\infty} \frac{\sum_{k=S}^{S+\left\lfloor\frac{(\tau-1)}{\bmod Q-i}\right\rfloor} \ell\left(k Q+H+i, \mathbf{x}^{\star}(i), \mathbf{u}^{\star}(i)\right)}{Q} \\
= & \lim _{S \rightarrow+\infty} \frac{\sum_{k=0}^{S-1} \ell\left(k Q+H+i, \mathbf{x}^{\star}(i), \mathbf{u}^{\star}(i)\right)}{Q} \\
= & \frac{\bar{\ell}\left(\mathbf{x}^{\star}(i), \mathbf{u}^{\star}(i)\right)}{Q}
\end{aligned}
$$

Remark 1 When analyzing asymptotic stability of periodic optimal solutions a suitable notion of dissipativity for an extended system can be adopted in the context of Economic MPC with time-invariant stage costs (see for instance Müller et al. (2015)). We believe that similar conditions can be adopted in the context of periodic stage costs given their 'time-invariance' for the period $T$ map. This is however outside the scope of the present manuscript.

\section{EMPC with parameter-varying costs}

We consider next the case of a stage cost taking the form (5), when transitions between different parameter values happen with a frequency that is comparable to the inverse of the process transients. In this case, it is meaningful to design terminal trajectories not only in correspondence to the individual best-steady states computed for frozen $\theta$ parameters, but also to consider connection orbits that serve the purpose of achieving an optimized transition between different best-operating regimes, while, at the same time, avoiding feasibility losses. For $i=1, \ldots, N$ we may define best steady-states as follows:

$$
\begin{aligned}
& \ell_{i}^{\star}:=\min _{x, u} \tilde{\ell}\left(\theta_{i}, x, u\right) \\
& \quad \text { subject to } \\
& x=f(x, u) \\
& \quad(x, u) \in \mathcal{Z} .
\end{aligned}
$$

We denote the corresponding best steady-state input pairs as $\left(x_{i}^{\star}, u_{i}^{\star}\right)$. At the same time, given $i \neq j$ in $\{1,2, \ldots, N\}$ we assume existence of feasible trajectories moving out of $x_{i}^{\star}$, reaching $x_{j}^{\star}$ in $\tau_{i j}>0$ steps. These trajectories are taken by solving the following optimiza- tion problem:

$$
\begin{aligned}
\ell_{i j}^{\star} & :=\min _{\mathbf{z}, \mathbf{v}} \tilde{\ell}\left(\theta_{i}, z(0), v(0)\right)+\sum_{k=1}^{\tau_{i j}-1} \tilde{\ell}\left(\theta_{j}, z(k), v(k)\right) \\
& \text { subject to } \\
& z(k+1)=f(z(k), v(k)) \\
& (z(k), v(k)) \in \mathcal{Z} \\
& z(0)=x_{i}^{\star} \\
& z\left(\tau_{i j}\right)=x_{j}^{\star} .
\end{aligned}
$$

Our main assumptions for this set-up will be:

- For each pair $(i, j)$ there exists a finite control sequence $\mathbf{u}_{i j}^{\star}$ able to steer the state from $x_{i}^{\star}$ to $x_{j}^{\star}$ in $\tau_{i j}$ steps. We denote by $\mathbf{x}_{i j}^{\star}$ the corresponding state sequence.

- Dwell-time: after switching between $\theta_{i}$ to $\theta_{j}$, at least $\tau_{i j}$ steps pass before another switching occurs.

The value of $x_{F}(t)$ is decided at each sampling time by a supervisory logic that assigns $x_{F}(t)=x_{i}^{\star}$, provided the current value of $\theta(t)$ is $\theta_{i}$. If, however, a switching is detected between $\theta_{i}$ and $\theta_{j}$, that is $\theta(t+H)=\theta_{j}$, (while $\theta(t+H-1)=\theta_{i}$, where $H$ denotes the control horizon, then a transition mode is enabled and the supervisor assigns $x_{F}(t+k)=\mathbf{x}_{i j}^{\star}(k)$ for the subsequent $\tau_{i j}$ time steps. Eventually, $x_{F}\left(t+\tau_{i j}+k\right)=x_{j}^{\star}$, and maintain such value until further notice, viz. until another switching is detected. Notice that this supervisory logic needs to know ahead of time the value of $\theta(t)$. Accordingly, we define the following indicator functions: $\chi_{i}(t)=1$ if and only if the supervisory logic is operating in standard mode with $\theta(t)=\theta_{i}\left(\chi_{i}(t)=0\right.$ otherwise $) ; \chi_{i j}(t)=1$ iff the supervisory logic is operating in transient mode during a switch between parameters $\theta_{i}$ and $\theta_{j}$. In order to carry out derivations it will also be useful to define the indicator function $\chi_{i j}(t, k)=1$ iff at time $t$ we are in the $k$-th step of the transient mode between parameters $\theta_{i}$ and $\theta_{j}$.

We may define the average of $\chi_{i}(t)$ and $\chi_{i j}(t)$ as given below:

$\bar{\chi}_{i}=\lim _{T \rightarrow+\infty} \frac{\sum_{t=0}^{T-1} \chi_{i}(t)}{T} \quad \bar{\chi}_{i j}=\lim _{T \rightarrow+\infty} \frac{\sum_{t=0}^{T-1} \chi_{i j}(t)}{T}$.

Under the stated assumptions, an apriori bound on the asymptotic performance may be obtained by considering that the average cost of $x_{F}(t)$ is given as from the following Lemma.

Lemma 3 Assume that parameter switching occurs according to the dwell-time constraint and that $x_{F}(t)$ is defined according to the supervisory logic described, then, if asymptotic averages of indicator functions $\chi_{i}$ and $\chi_{i j}$ 
exist, the following holds:

$\lim _{\tau \rightarrow+\infty} \frac{\sum_{t=0}^{\tau-1} \ell\left(t+H, x_{F}(t), u_{F}(t)\right)}{\tau}=\sum_{i} \bar{\chi}_{i} \ell_{i}^{\star}+\sum_{i \neq j} \bar{\chi}_{i j} \frac{\ell_{i j}^{\star}}{\tau_{i j}}$.

Proof: Notice that, by definition:

$$
1=\sum_{i=1}^{l} \chi_{i}(t)+\sum_{i \neq j} \chi_{i j}(t), \quad \forall t \in \mathbb{N} .
$$

Moreover,

$$
\chi_{i j}(t)=\sum_{k=0}^{\tau_{i j}-1} \chi_{i j}(t, k) .
$$

Then, exploiting equation (17) yields:

$$
\begin{aligned}
\sum_{t=0}^{\tau-1} \ell\left(t+H, x_{F}(t), u_{F}(t)\right)= & \sum_{t=0}^{\tau-1}\left(\sum_{i=1}^{l} \chi_{i}(t)+\sum_{i \neq j} \chi_{i j}(t)\right) \ell\left(\theta_{\sigma(t+H)}, x_{F}(t), u_{F}(t)\right) \\
= & \sum_{t=0}^{\tau-1}\left(\sum_{i=1}^{l} \chi_{i}(t) \ell\left(\theta_{\sigma(t+H)}, x_{F}(t), u_{F}(t)\right)+\right. \\
= & \sum_{t=0}^{\tau-1}\left(\sum_{i=1}^{l} \chi_{i}(t) \ell\left(\theta_{i}, x_{i}^{\star}, u_{i}^{\star}\right)+\right. \\
\left.\sum_{i \neq j} \sum_{k=0}^{\tau_{i j}-1} \chi_{i j}(t, k) \ell\left(\theta_{\sigma(t+H)}, x_{F}(t), u_{F}(t)\right)\right) & \sum_{k=1}^{\tau-1}\left(\sum_{i=0}^{l} \chi_{i j}(t) \ell\left(\theta_{i}, x_{i}^{\star}, u_{i}^{\star}\right)+\sum_{i \neq j}\left(\chi_{i j}(t, k) \ell\left(\theta_{j}, \mathbf{x}_{i j}^{\star}(k), \mathbf{u}_{i j}^{\star}(k)\right)\right)\right. \\
= & \left.\sum_{i=1}^{\tau_{i j}-1}, \mathbf{x}_{i j}^{\star}(0), \mathbf{u}_{i j}^{\star}(0)\right)+
\end{aligned}
$$

Then, by dividing the previous expression by $\tau$ and letting $\tau \rightarrow+\infty$, one has:

$$
\begin{aligned}
& \lim _{\tau \rightarrow+\infty} \frac{\sum_{t=0}^{\tau-1} \ell\left(\theta_{\sigma(t+H)}, x_{F}(t), u_{F}(t)\right)}{\tau} \\
& =\lim _{\tau \rightarrow+\infty} \frac{\sum_{t=0}^{\tau-1}\left(\sum_{i=1}^{l} \chi_{i}(t) \ell\left(\theta_{i}, x_{i}^{\star}, u_{i}^{\star}\right)\right)}{\tau} \\
& +\lim _{\tau \rightarrow+\infty} \frac{\sum_{t=0}^{\tau-1}\left(\sum_{i \neq j} \sum_{k=0}^{\tau_{i j}-1} \chi_{i j}(t, k) \ell\left(\theta_{j}, \mathbf{x}_{i j}^{\star}(k), \mathbf{u}_{i j}^{\star}(k)\right)\right)}{\tau} \\
& =\sum_{i=1}^{l} \bar{\chi}_{i} \ell_{i}^{\star}+\sum_{i \neq j} \frac{\bar{\chi}_{i j}}{\tau_{i j}} \ell_{i j}^{\star} .
\end{aligned}
$$

where the last equality follows by considering that:

$$
\lim _{\tau \rightarrow+\infty} \frac{\sum_{t=0}^{\tau-1} \chi_{i j}(t, k)}{\tau}=\frac{\bar{\chi}_{i j}}{\tau_{i j}}
$$

Application of Lemma 3, together with Lemma 1, allows to have an a priori upper-bound for the asymptotic average performance of the closed-loop system in relation to the terminal ingredients and transient trajectory costs designed in the formulation of the economic MPC algorithm as well as the switching signal $\theta(t)$.

Remark 2 It is known that asymptotic stability of Economic MPC can be studied by making reference to suitable dissipativity notions. As shown in the Example considered in the following Section, the solution $x_{F}(t)$ obtained by collating equilibrium solutions with transient reconciling trajectories is not generally expected to be optimal if cost functionals are persistently varying in time (for instance in a periodic fashion). Hence, even if individual equilibrium states may fulfill a dissipativity property when considered in a time-invariant context with their associated stage-cost, there is no reason why asymptotic stability of the $x_{F}(t)$ solution should be expected in closed-loop. For signals $\theta(t)$ which are eventually constant and equal to $\theta_{i}$, asymptotic stability of the associated equilibrium follows provided strict dissipativity holds for some storage function $\lambda$ with respect to the supply function $\ell\left(\theta_{i}, x, u\right)-\ell\left(\theta_{i}, x_{i}^{\star}, u_{i}^{\star}\right)$.

\section{Simulation results}

In this section the control of a nonlinear isothermal chemical reactor with consecutive-competitive reactions, see Lee \& Bailey (1980), has been dealt with. Such reactions arise in many chemical and biological applications. In the simple case of two reactions, the following structure should be taken into account

$$
\begin{aligned}
& P_{0}+B \rightarrow P_{1} \\
& P_{1}+B \rightarrow P_{2}
\end{aligned}
$$

The dimensionless mass balances for this problem are

$$
\begin{aligned}
& \dot{x}_{1}=u_{1}-x_{1}-\sigma_{1} x_{1} x_{2} \\
& \dot{x}_{2}=u_{2}-x_{2}-\sigma_{1} x_{1} x_{2}-\sigma_{2} x_{2} x_{3} \\
& \dot{x}_{3}=-x_{3}+\sigma_{1} x_{1} x_{2}-\sigma_{2} x_{2} x_{3} \\
& \dot{x}_{4}=-x_{4}+\sigma_{2} x_{2} x_{3}
\end{aligned}
$$

where $x_{1}, x_{2}, x_{3}$ and $x_{4}$ are the concentrations of $P_{0}$, $B, P_{1}$ and $P_{2}$ respectively, while $u_{1}$ and $u_{2}$ are the inflow rates of $P_{0}$ and $B$ and represent the manipulated 

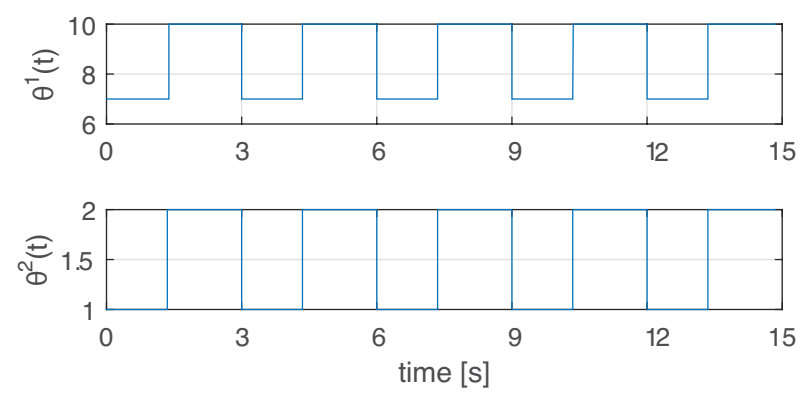

Fig. 1. time-varying parameters for the cost (19)
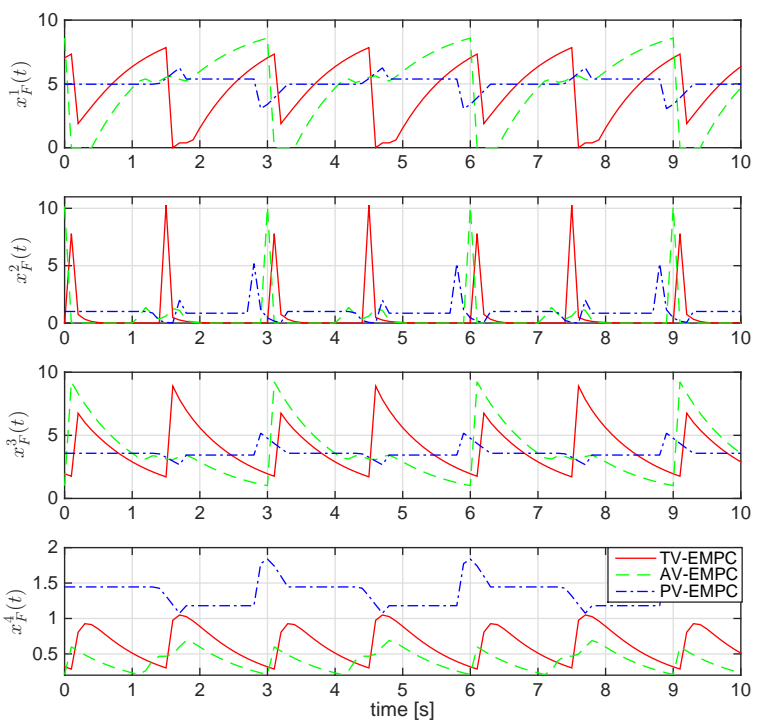

Fig. 2. Precomputed solutions for each algorithm: best $T$-Periodic solutions for the TV-EMPC, best $Q$-Periodic solution for the $A V-E M P C$ and switching solutions for the PV-EMPC
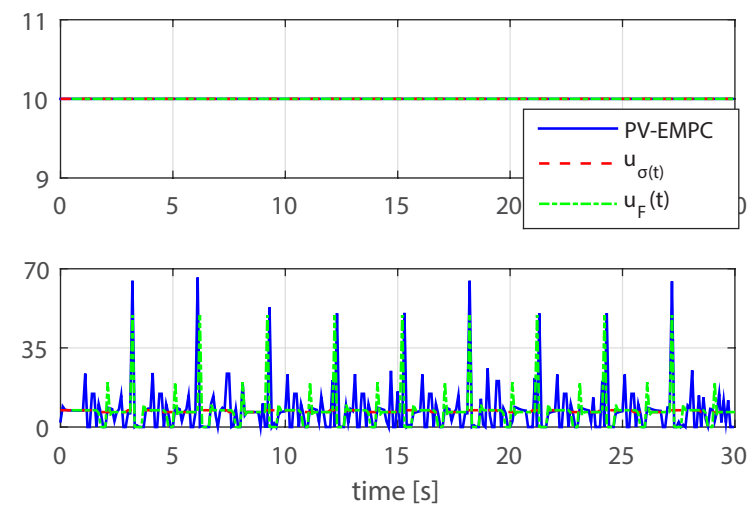

Fig. 3. Input Commands in the case of the PV-EMPC

variables. The parameters $\sigma_{1}$ and $\sigma_{2}$ take, in the following simulations, the values 1 and 0.4 respectively. An upper bound of 10 is imposed on $u_{1}$. The simultaneous
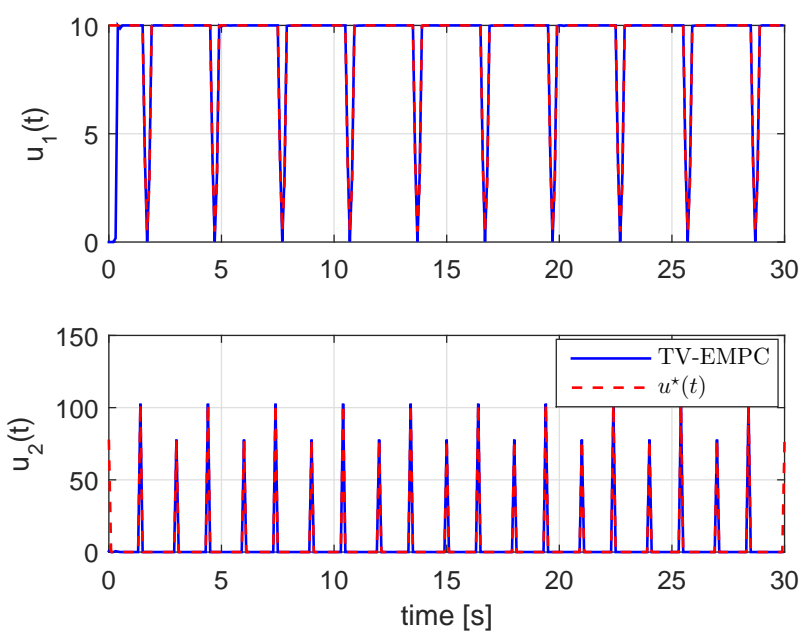

Fig. 4. Input Commands in the case of the TV-EMPC
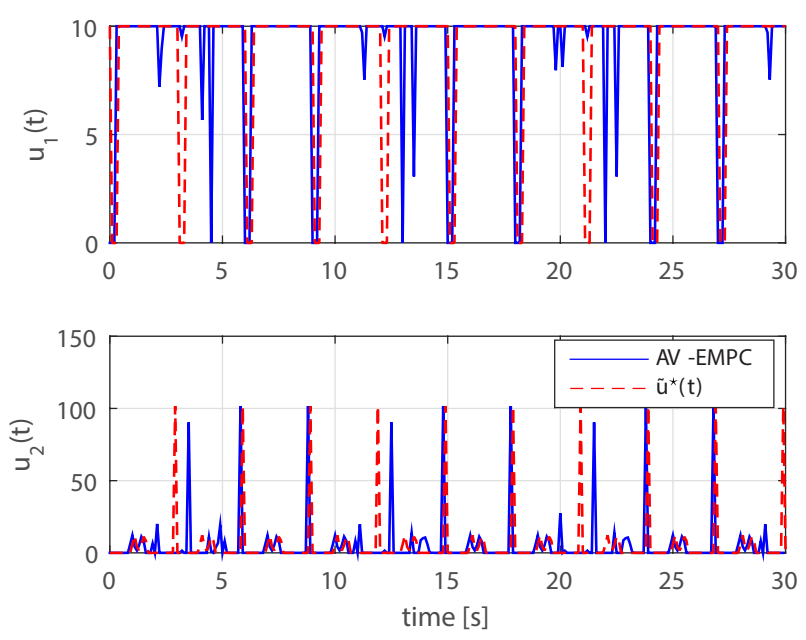

Fig. 5. Input Commands in the case of the AV-EMPC

approach of Flores-Tlacuahuac et al. (2008) has been used to solve the dynamic regulation problem. The statespace is divided into a fixed number of finite elements. The input is parameterized according to zero-order hold with the input value constant across a finite element. A sampling time $T_{s}=0.1 s$ has been chosen. The system is managed according to the following economic cost function that is based on two configurations of the price of product $P_{1}$ and flow rate of $P_{0}$ and $B$

$$
\ell(t, x, u)=-\theta_{i}^{1}(t) x_{3}+\theta_{i}^{2}(t) u_{1}+\theta_{i}^{2}(t) u_{2}
$$

where the parameters $\theta_{i}=\left(\theta_{i}^{1}, \theta_{i}^{2}\right), i=1,2$ are the prices per unit of product for the $i$-th configuration. It is assumed that the cost parameters periodically varies between the cost configuration $\theta_{1}=(7,1), \theta_{2}=(10,2)$ following the trend of a discrete-time square wave with 


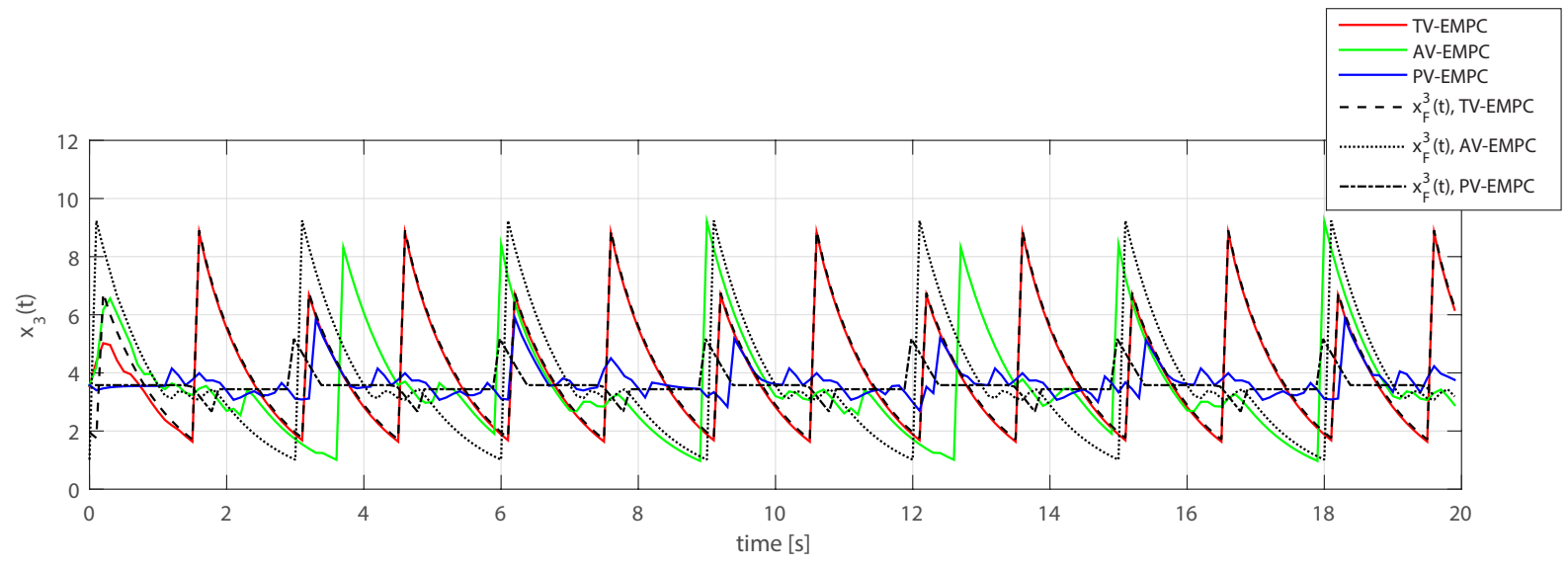

Fig. 6. Comparison on evolution of $x_{3}(t)$

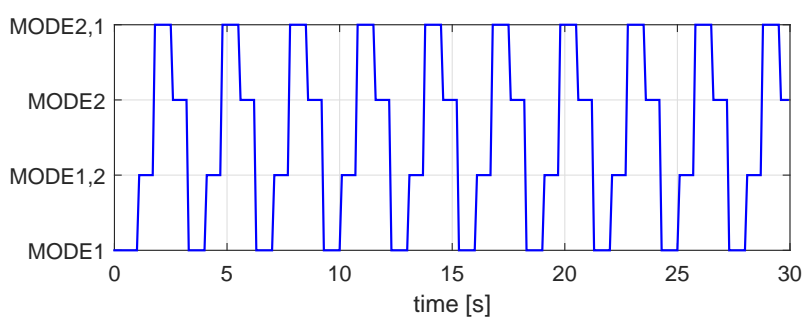

Fig. 7. Active modes of the S-Logic in the case of PV-EMPC

period $T=30$ (see Figure 1$)$. As a consequence, the averaged cost $\bar{\ell}$ takes the following form

$$
\bar{\ell}(x, u)=-\frac{\theta_{1}^{1}+\theta_{2}^{1}}{2} x_{3}+\frac{\theta_{1}^{2}+\theta_{2}^{2}}{2} u_{1}+\frac{\theta_{1}^{2}+\theta_{2}^{2}}{2} u_{2}
$$

In this example the above presented EMPC based schemes have been compared. For the sake of clarity we denote with the acronym TV-EMPC the scheme based on the best periodic solution (12), while the scheme associated to the averaged solution (13) is here referred as AV-EMPC. Finally the acronym PV-EMPC will identify the EMPC with parameter-varying cost described in Section 4. In this respect, we have first computed by means of (15) the economically optimal steadystate conditions associated to each cost configuration obtaining: $x_{1}^{\star}=[4.9759,1.0097,3.5787,1.4454]^{T} u_{1}^{\star}=$ $[10,7.4791]^{T}, x_{2}^{\star}=[45.3813,0.8583,3.4383,1.1804]^{T}$ $u_{2}^{\star}=[10,6.6573]^{T}$, with related steady-state cost $\ell_{1}^{\star}=-7.57$ and $\ell_{2}^{\star}=-1.07$ respectively. From these solutions, two reconciliating sequences $\mathbf{x}_{i j}, i \neq j$ have been derived (see Figures 2 (blue dash-dotted line) and 3 (green dash-dotted line)) for the PV-EMPC by exploiting (16), along with related dwell times $\tau_{12}=\tau_{21}=8$ and associated transient costs $\frac{\ell_{12}^{\star}}{\tau_{12}}=-2.58$ and $\frac{\ell_{21}^{\star}}{\tau_{21}}=-7.14$. Secondly, we have solved problem (12) and computed the best $T$-periodic solutions for the TV-EMPC algorithm and obtained the minimum cost $\ell_{T}^{\star}=-15.81$ with solutions depicted in Figures 2 (black dashed line) and 4 (red dashed line). Thirdly, a $Q$-periodic solution for the AV-EMPC algorithm has been evaluated for $Q=30$ from (13) obtaining the minimum averaged cost $\bar{\ell}_{Q}^{\star}=-9.94$ and $Q$-periodic solutions depicted in Figures 2 (black dotted line) and 5 (red dashed line).

The prediction horizon has been set to $N=5$. The initial condition is $x(0)=x_{1}^{\star}$. A simulation horizon of $30 s$ is chosen with sampling time $T_{s}$.

The system behavior along the simulation has been plotted in the Figures 3-6 while in Figure 7 the active modes for the PV-EMPC have been illustrated. All the controllers give rise to an oscillating solution. This aspect is expected for both TV-EMPC and AV-EMPC and it is not surprising for the PV-EMPC because, from a performance point of view, its "oscillating behavior" represents an economic benefit with respect to a "converging behavior" to a steady state solution ((Angeli et al., $2012)$ ). In this respect in order to compare the behavior of closed-loop systems, the average profit, that is $A v\left[\theta_{i}^{1} x_{3}-\theta_{i}^{2} u_{1}-\theta_{i}^{2} u_{2}\right]$, has been evaluated and compared in Table I. It is evident that the strategies based on pre-computed terminal constraints (TV-EMPC, AVEMPC) present better performance with respect to the PV-EMPC.

\begin{tabular}{|c||c||c|}
\hline Case & Avg. cost & Apriori upper bound \\
\hline TV-EMPC & -16.47 & $\ell_{30}^{\star}=-15.81$ \\
\hline AV-EMPC & -10.02 & $\bar{\ell}_{40}^{\star}=-9.94$ \\
\hline PV-EMPC & -5.93 & $\begin{array}{l}\frac{7}{30}\left(\frac{\ell_{12}^{\star}}{\tau_{12}}+\frac{\ell_{21}^{\star}}{\tau_{21}}\right) \\
+\frac{8}{30}\left(\ell_{1}^{\star}+\ell_{2}^{\star}\right)=-4.6\end{array}$ \\
\hline
\end{tabular}

Table 1

Average Profit for the Closed-Loop profiles 


\section{Conclusions}

This paper illustrates and interprets recent advances in time-varying Economic Model Predictive Control. In particular, it deals with the case of time-varying costs, both in the case of a priori known time-varying costs (periodic or admitting an asymptotic average) and in the case of parameter varying costs. The different solutions and performance bounds are compared by means of a simple case study where all the different approaches have been tested. The question of how dissipativity may affect stability and convergence of solutions to the terminal constraints $x_{F}(t)$ is still open, although, we expect some form of these conditions to be applicable, at least in the case of periodic stage costs and/or eventually constant LPV scenarios.

\section{References}

Adeodu, O., \& Chmielewski, D. J. (2013). Control of electric power transmission networks with massive energy storage using economic mpc. In American Control Conference $(A C C)$, 2013. Washington DC.

Angeli, D., Amrit, R., \& Rawlings, J. B. (2012). On average performance and stability of economic model predictive control. IEEE Transactions on Automatic Control, 57 (7), pp. 1615-1626.

Angeli, D., Casavola, A., \& Tedesco, F. (2015a). Economic model predictive control with parametervarying cost and guaranteed average performance. In Proc. of the 54th IEEE Control and Decision Conference. Osaka, Japan.

Angeli, D., Casavola, A., \& Tedesco, F. (2015b). On average performance of economic model predictive control with time-varying cost and terminal constraints. In American Control Conference ( $A C C)$. Chicago, IL.

Angeli, D., Casavola, A., \& Tedesco, F. (2015c). Theoretical advances on economic model predictive control with time-varying costs. In 5th IFAC Conference on Nonlinear Model Predictive Control NMPC 2015. Seville, Spain.

Cole, W. J., Morton, D. P., \& Edgar, T. F. (2014). Optimal electricity rate structures for peak demand reduction using economic model predictive control. Journal of Process Control, 24 (8), pp. 1311-1317.

Ellis, M., \& Christofides, P. D. (2014). Economic model predictive control with time-varying objective function for nonlinear process systems. AIChE Journal, 60 (2), pp. 507-519.

Ferramosca, A., Limon, D., \& Camacho, E. F. (2014). Economic mpc for a changing economic criterion for linear systems. IEEE Trans. on Automatic Control, 59 (10), pp. 2657-2667.

Flores-Tlacuahuac, A., Moreno, S. T., \& Biegler, L. T. (2008). Global optimization of highly nonlinear dynamic systems. Industrial $\&$ Engineering Chemistry Research, 47 (8), pp. 2643-2655.
Gopalakrishnan, A., \& Biegler, L. T. (2013). Economic nonlinear model predictive control for periodic optimal operation of gas pipeline networks. Computers $\&$ Chemical Engineering, 52, pp. 90-99.

Grosso, J., Ocampo-Martinez, C., Puig, V., Limon, D., \& Pereira, M. (2014). Economic mpc for the management of drinking water networks. In 13th European Control Conference (ECC), 2014. Strasbourg, France.

Hovgaard, T. G., Edlund, K., \& Bagterp Jorgensen, J. (2010). The potential of economic mpc for power management. In 49th IEEE Conference on Decision and Control $(C D C)$, 2010. Atlanta, GA.

Huang, R., Biegler, L. T., \& Harinath, E. (2012). Robust stability of economically oriented infinite horizon nmpc that include cyclic processes. Journal of Process Control, 22 (1), pp. 51-59.

Lee, C., \& Bailey, J. E. (1980). Modification of consecutive-competitive reaction selectivity by periodic operation. Industrial \& Engineering Chemistry Process Design and Development, 19 (1), pp. 160-166.

Limon, D., Pereira, M., Muñoz de la Peña, D., Alamo, T., \& Grosso, J. (2014). Single-layer economic model predictive control for periodic operation. Journal of Process Control, 24 (8), pp. 1207-1224.

Ma, J., Qin, S. J., \& Salsbury, T. (2014). Application of economic mpc to the energy and demand minimization of a commercial building. Journal of Process Control, 24, pp. 1282-1291.

Müller, M. A., Grüne, L., \& Allgöwer, F. (2015). On the role of dissipativity in economic model predictive control. In 5th IFAC Conference on Nonlinear Model Predictive Control NMPC 2015. Seville, Spain.

Touretzky, C. R., \& Baldea, M. (2014). Integrating scheduling and control for economic mpc of buildings with energy storage. Journal of Process Control, 24 (8), pp. 1292-1300.

David Angeli received the Laurea and Ph.D. degrees from the University of Florence, Italy, in 1996 and 2000, respectively. He was later appointed Assistant Professor within the Department of Systems and Computer Science of the same University and Associate Professor since 2005. He is currently a Reader in Stability of Nonlinear Systems at Imperial College London after joining the Department of Electrical and Electronic Engineering in 2008. His research interests include: stability and dynamics of nonlinear systems and networks, control of constrained systems and optimization, biomolecular dynamics and control solutions for the smart grid. He is author of more than 80 Journal publications in the field of Automatic Control and has served as an Associate Editor of IEEE Transactions on Automatic Control and Automatica. He is an IEEE Fellow since 2015.

Alessandro Casavola was born in Florence, Italy, in 1958. He received the Ph.D degree in Systems Engineering from the University of Bologna, Italy, in 1990. He has been with the Department of Systems and Computer Engineering of the University of Florence from 1996 to 
1998 as a Researcher. Since 1998 he is with the Department of Computer Science, Modeling, Electronics and Systems Engineering of the University of Calabria: as an Associate Professor first and as Full Professor since 2005. His current research interests include constrained predictive control, control under constraints, control reconfiguration for fault tolerant systems and supervision approaches over data networks. His is a Subject Editor of the International Journal of Adaptive Control and Signal Processing.

Francesco Tedesco was born in Cosenza, Italy, in 1984. He received the Laurea Specialistica degree in Automation Engineering in 2008 and his Ph.D. in Systems and Computer Engineering in 2012 from the University of Calabria, Italy. Since 2012 he is an Assistant Researcher at the DIMES department of the University of Calabria. His main research interests involve supervision approaches over data network, Model Predictive Control, automotive applications and control of power systems. 